\title{
A Conflict Resolution Strategy Selection Method (ConfRSSM) in Multi-Agent Systems
}

\author{
Alicia Y.C. Tang \\ College of Computer Science and Information Technology \\ Universiti Tenaga Nasional \\ 43000 Kajang Selangor Malaysia
}

\author{
Ghusoon Salim Basheer \\ College of Graduate Studies \\ Universiti Tenaga Nasional \\ 43000 Kajang Selangor Malaysia
}

\begin{abstract}
Selecting a suitable conflict resolution strategy when conflicts appear in multi-agent environments is a hard problem. There is a need to formulate a model for strategic decision making in selecting a strategy to resolve conflicts. In this paper, we formalise a model for selecting a conflict resolution strategy in multi-agent systems. The model is expected to select a suitable strategy which guaranties low cost in terms of the number of messages and time ticks. This paper focuses on a novel method to guide strategic decision making for conflict resolution. The proposed model is named as Conflict Resolution Strategy Selection Method (ConfRSSM). We identified three distinct types of intervention: (1) domain requirement, (2) conflict strength, and (3) confidence level of the conflicting agents. We also ascertain that the most appropriate conflict resolution strategy for a given conflict depends on the type of conflict (weak, strong), the agents' confidence level, and the domain preferences. Our method explores the best strategic choices that will reduce the cost and time in selecting a strategy.
\end{abstract}

Keywords-Conflict resolution; Confidence level; Multi-agent system; Strategy selection method

\section{INTRODUCTION}

When agents work as a team, in the environments of multiagent, and a conflict state appears among them, there is a need to select one of the multiple strategies to resolve the conflict. Equipping agents with the capability to choose one or more strategies gives them more flexible behaviour [1]. Results of previous research on various conflict resolution strategies provide a foundation to solve the conflict problem, but there is very limited research focusing on how agents should select the most appropriate conflict resolution strategy given the goals and current situational context [2, 3, 4]. A major characteristic of most conflict resolution strategy approaches for multi-agent conflict resolution is that they focused on negotiation, arbitration, or other strategy, but not considering the characteristic of conflict states, or the confidence levels of conflicting agents' opinions.

On the other hand, some popular conflict resolution strategies are suffering from several weaknesses. As mentioned by Barber et al. [1], if there is more than one proposal within negotiation strategy, the number of required messages is much more than any other strategy, which makes reaching an agreement state more complicated. If the message has high cost bandwidth, this makes negotiation a high cost strategy. Additionally, heavy coordination between agents can be a cause of communication bottleneck that has bad effects on scalability and robustness of the system. If any conflict state among agents resolved by negotiation strategy that requires many messages, this may lead to a heavy coordination state. Based on this introduction, there is a real need to include some strategies that ignore some unimportant conflict states or include submitting strategy to enhance the performance the conflict resolution method.

Current conflict resolution literature on resolving conflict is deficient in four major areas: (1) There is no clear attention to a confidence level of conflicting agents' opinions and the effects of these levels on the conflict outcome, (2) There is no attention to the number of conflicting agents (number of groups) and number of issues that agents (groups) conflicts about it, (3) There is no suggestion of ignoring some unimportant conflict states using submitting strategy to enhance the conflict resolution process, and (4) There are no rules to select an appropriate strategy to solve conflicts that guaranty less cost and time.

We will discuss a new method for selecting an optimal strategy to resolve conflicts. We argue that conflict resolution strategies in multi-agent systems need to simulate the resolution of conflict in real life. We proposed that an agent must have ability to select an appropriate conflict resolution strategy, according to: the strength of conflicts (e.g. Weak conflict, or strong conflict), the agent's confidence level in their opinions (e.g. High level opinion's confidence, and low level opinion's confidence).

\section{RELATED WORK}

Conflict resolution strategies in multi-agent systems need to simulate the natural resolution of conflict in real life. Results of previous research on various conflict resolution strategies do provide a foundation to solve the conflict problem, but there is limited research focusing on how agents should select the most appropriate conflict resolution strategy given the goals and current situational context. Most state-of-the-art techniques have not considered all the possible states of conflict occurrences $[5,6]$.

\section{A. Conflicts in Multi-agent Systems}

A multi-agent system is considered as a collection of entities communicating and interacting with each other to achieve individual or collective goals. However, agents occasionally overlook the total view of the overall problem, which causes conflicts among them [7]. A conflict is any situation of disagreement between two or more agents or two 
or more groups of agents. This disagreement can be in plans, desires, or belief.

Conflict between agents arises in a multi-agent environment in many cases, and it is solved depending on its type and dimension. Tessier [8] classified conflicts into two main classes: physical conflicts and knowledge conflicts. Physical conflicts are consequences of external and resource conflicts. Knowledge conflicts (or epistemic conflicts) occur when each agent has its own information that is different from other agents. In this class of conflict, agents conflict in beliefs, knowledge and opinions.

Inspired from human's conflict resolution strategies, we presented a framework for conflict resolution [9], as follows:

- Forcing: corresponds to Destruction in some conflict state. We recognise that there is no chance to resolve the conflict.

- Submitting/Ignoring: corresponds to Subservience. In this case, there is no force, but inducement between both sides.

- Delegation: corresponds to Delegation when the conflict cannot be resolved, both opponents request a third party that has deep knowledge to judge.

- Negotiation: corresponds to Compromising through negotiation when one of the opponents is willing to yield. This state includes an agreement in a different style.

- Agreement: corresponds to Consensus. Each opponent must give all details about its decision to a third party. For this reason, this process comes as a result of a delegation process.

\section{B. State-of-the-art in Conflict Resolution}

Knowing the nature of a conflict reduces the search space of possible resolution strategies and thus helping agents to select the most appropriate behaviours that are most effective to resolve the conflict [1]. From literature, there are many different approaches associated with conflict resolution strategies, but the important question is how an agent selects the most suitable strategy for its situation and aims. Liu et al. [3] argued that agents should select an appropriate strategy for conflict resolution depending on three factors: type of conflict, agent's rule, and preference solution. They classified conflicts into three classes: goal conflicts, plan conflicts, and belief conflicts. After classifying conflicts that appeared in the system, many modifications such as goal modification, plan modification, and desire modification are performed to resolve the conflicts. Adler et al. [4] allowed an agent to select a specific strategy from many other strategies such as priority agreement, negotiation, arbitration, and self-modification.

\section{Conflict Resolution Strategy Selection}

The capability of strategy selection can enhance multiagent systems' flexibility and adaptability to dynamic and uncertain environments. For instance, when a conflict occurs in distributed agents over shared resources, we need a strategy that distributes resources equally among all agents, or a strategy that offers maximum possible resources to most constrained agents [10]. Few researchers discussed the ability of agents to switch between multiple conflicts strategies [3, 6, 10]. To achieve an appropriate selection of conflict resolution strategy, several issues need to be addressed:

- A uniform representation of different strategies to assist the comparison and evaluation process;

- A metal-level reasoning mechanism for strategic decision making;

- A set of specifications (including domain requirements) that agents use to evaluate alternative strategies;

- Adaptability to support the decision-making required to select a strategy.

In the selection of a conflict resolution strategy, Barber et al. [1] raised the issue of whether the domain's requirements satisfied by the selected strategy. For example, an agent might use the high cost strategy in a domain that requires minimum cost. There is also the important issue of the confidence level of agents' opinions that affects the selection of appropriate strategy. Barber's research demonstrated one approach for matching four resolutions strategies (Negotiation, Arbitration, Self-Modification and Voting) [1, 2].

\section{Limitations}

Most work did not exploit other information such as the number of conflicting agents, confidence level of the agents and conflict strengths. According to Thomas [11], it is hard to select an appropriate strategy without having the information about an agent's confidence level. To provide a near-perfect method of a conflict resolution strategy selecting operation, the strength of conflict and the confidence level of agents need to be analysed. Our argument for such proposition is that we should not ignore the influence of the confidence levels of conflicting agents that control the direction of conflict resolution processing. The agents' confidence levels are important since a high confidence level may lead to selecting a forcing or any strategies.

\section{RESEARCH BACKGROUND AND FOCUS}

The main objective of our research is to develop an integrated framework that comprised of Agent Confidence Detection Technique (AgConfDT) that detects agent's confidence levels, and a Conflict Strength Detection Model (CSDM) that detects conflict strength. This information is used by a Conflict Resolution Strategy Selection Method (ConfRSSM) for selecting a suitable conflict resolution strategy. AgConfDT includes an exploration of the three different confidence factors (trust, certainty, and evidences) [12]. It emphasises important objects by integrating these factors in order to better understand the agents' specifications since the technique can detect the agent's confidence whenever in the absence of any required information. Results show that the proposed technique eliminates untested opinions, such that the confidence levels of conflicting agents can be detected in all cases although in the absence of some confidence factors. CSDM detects the disagreement degree among the conflicting 
agents, a conflict ratio as input for the model, and the output is the conflict strength.

In resolving a conflict, ConfRSSM uses the confidence levels of conflicting agents and a conflict strength to select a suitable strategy. We hypothesise that ConfRSSM can reduce the number of messages and time ticks by ignoring some unimportant conflict states, which increases the efficiency of the entire conflict resolution process.

The main research activities of the integrated framework for ConfRSSM are summarised as follows:

\section{A. Developing Agent Confidence Level Detection Technique}

In the work described in [12], we define "confidence" as a combined model that considers social trust and certainty concepts, supported by collecting evidence. We have a decision to be decided depending on collecting agents' opinions; a confidence value used for each agent to resolve any opinion conflict. Modelling confidence based on three sources of information, which are the degree of certainty regarding the opinion of each agent, agent's trust, and evidence for both certainty and trust. We combine trust and certainty values into a single composite measure to integrate a holistic view of the confidence of an agent. The concept of confidence is decomposed into several factors, which may be integrated to produce the final confidence evaluation (degree of confidence). Figure 1 shows an illustration of the interaction between Evidential Agent (EA) and Evaluation Agent (EVA) in the confidence mode. EVA Collects evidences from the environment. EA is responsible for calculating agents' confidence levels. One of the main specifications of our design is the assumption that the EA depends on the opinions of other agents to make its decision. Thus, the EA can have more confidence in some agents than others, which could change based on evidence. In order to process these evidences we introduce an EVA. Here, we include evidence as an additional factor that sets the confidence values of agents. Assuming positive evidence for opinions matching agent I's certainty and trust, then it can be said that confidence increases as I's opinion matches the belief of the EA.

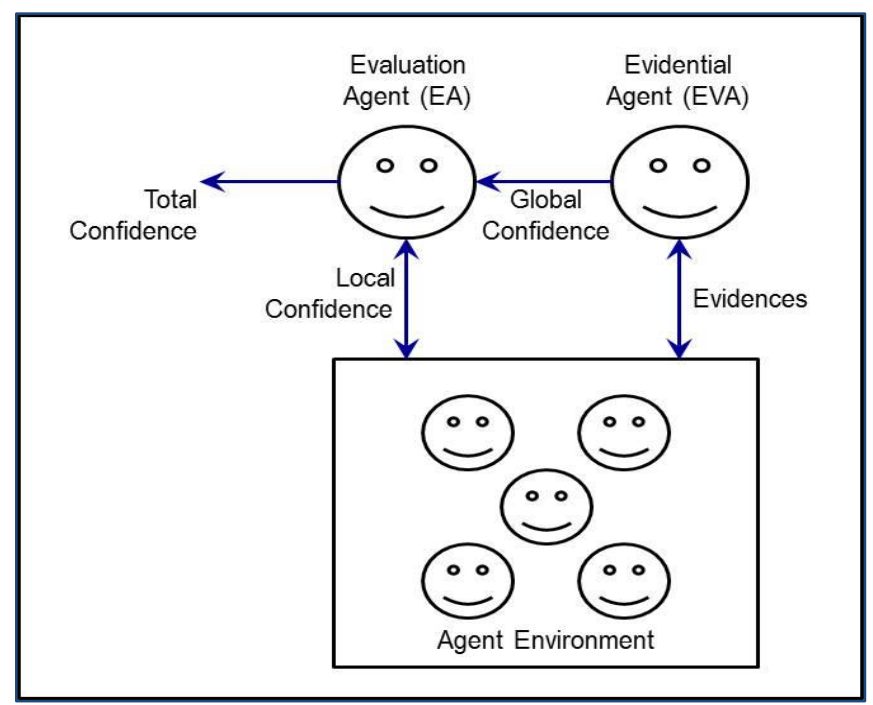

Fig. 1. Confidence Detection Model

\section{B. Developing a Classification Model for Conflict States}

Classification of conflicts provides a form of control in an environment in which agents are in conflicts with other agents in unknown conflict ratio and disagreement degree. Classification can be utilised to select the most appropriate resolution strategies to resolve conflicts rather than using one strategy in all conflict situations. For this purpose, we adapt a conflict model in which we define a conflict strength to be a particular measure of conflict between unknown numbers of agents about undefined dissenting issues [13]. Figure 2 depicts the analytical process of classifying the two dimensions of conflict resolution model.

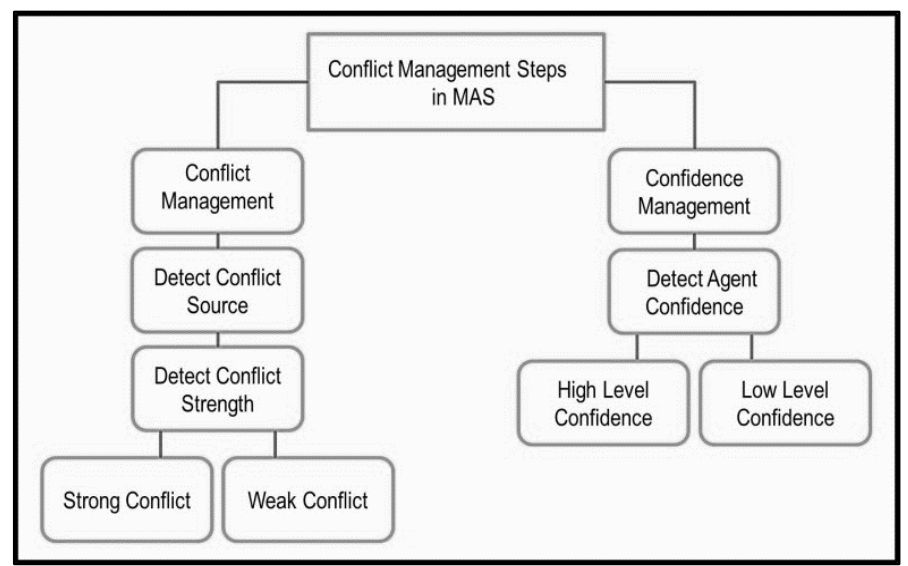

Fig. 2. A Model for Classifying Conflicts and Confidence in Multi-agent Systems

\section{Towards Developing a Conflict Resolution Strategy Selection Method}

After reviewing conflict resolution strategies in social science, we choose five strategies to resolve conflicts in our framework (Negotiation, Ignoring, Arbitration, Forcing, and Submitting). Efficient conflict resolution strategies mean resolving conflicts with fewer actions, and minimising the expected penalty [14]. From the review of current research work, there is no one strategy that works best for all situations. The following conflict states aspects are the focus of this research:

- Weak conflict versus strong conflict,

- Agents with high level confidence versus agents with low confidence level,

- Belief (opinion) conflict resolution,

- Agents' confidence levels effect on the selecting conflict resolution strategy,

- Conflict strengths effect on selecting a conflict resolution strategy,

- Conflict resolution strategy selection method.

Classifying conflicts into weak and strong is useful, and most importantly that, classifying agents based on their confidence level leads to wisdom selection of conflict resolution strategy. Conflicts among agents appeared when agents' opinions about one or multiple issues are different. In 
this situation, the conflict states classified into weak conflict and strong conflict based on the Conflict Ratio (CR) and Disagreement Degree (DD). CR is a ratio of conflicting agents to total number of agents, while DD is a ratio of dissenting issues to total number of issues in one conflicting state. Weak conflict means that result of adding DD with CR is less than one while strong conflict means that result of adding DD with $\mathrm{CR}$ is equal or more than one.

The next section provides the building blocks for the formulation of ConfRSSM.

\section{FORMALISING CONFRSSM DESIGN COMPONENTS}

The first important challenge in the field of agent's conflict is the question of how to select a suitable conflict resolution strategy. The second important aspect is the effect of confidence level of a conflicting agent on this selection. Efficient conflict resolution strategies mean resolving conflicts with fewer actions, and minimising the expected penalty [15]. At the very beginning of the strategic selection process, there are multiple strategies and there is a need to select just one. In order to understand the issues of conflicts in multi-agent environments, we analysed the social theory of conflict and propose a conflict resolution strategy.

\section{A. Definitions of ConfRSSM Components}

Definition 1: A set of agents $A$, each agent can represent as a tuple $\left(\mathrm{a}_{\mathrm{n}}, \mathrm{O}_{\mathrm{an}}(\mathrm{I})\right.$, Conf $\left._{\mathrm{an}}\right)$, Where:

$$
\begin{aligned}
& \mathrm{a}_{\mathrm{n}} \text { : any agent } \in \mathrm{A} \\
& \text { Conf }_{\mathrm{an}} \text { : an agent's confidence } \\
& \mathrm{O}_{\mathrm{an}}(\mathrm{I}) \text { : is the opinion of agent a of issue } \mathrm{I} \text {. }
\end{aligned}
$$

Definition 2: A conflict situation, $\mathrm{CS}$, is a state that occurs when an action performed by an agent objection by another agent, or when there is a disagreement state between two agents' opinions (decisions). Let us assume that there is a finite set of agents called the universe U. Elements of $U$ will be referred to as agents. We define Opinions Collection Function as follows:

Opinions Collection Function (OCF): This function collects an agent's opinion from the environment.

$$
\text { OCF }: U \rightarrow\left\{\mathrm{O}_{1} \ldots \mathrm{O}_{\mathrm{n}}\right\},
$$

Where:

$\mathrm{O}$ : is an agents' opinions

As mentioned in Definition 1, each agent in $U$ can be defined as a tuple $\left(\mathrm{a}_{\mathrm{i}}, \mathrm{O}_{\mathrm{ai}}(\mathrm{I}), \mathrm{Conf}_{\mathrm{ai}}\right)$. If there are any two agents $\left(\mathrm{a}_{\mathrm{i}}, \mathrm{O}_{\mathrm{ai}}(\mathrm{I}), \operatorname{Conf}_{\mathrm{ai}}\right)$, and $\left(\mathrm{a}_{\mathrm{j}}, \mathrm{O}_{\mathrm{aj}}(\mathrm{I}), \operatorname{Conf}_{\mathrm{aj}}\right)$, in $\mathrm{U}$, then, the conflict state appears if Oai $(\mathrm{I}) \neq \mathrm{O}_{\mathrm{aj}}(\mathrm{I})$.

The pair $C S=\left(a_{i}, a_{j}, I\right)$ represents a conflict situation, where $I$ is an issue that agents conflicts about.

Definition 3: A conflicting agents set, CAS, is a set of pairs of conflicting agents (or conflicting groups of agents). For example, if ai conflicts with aj, then CAS $=\{($ ai, aj) $\}$.

\section{B. Conflict Classification}

Conflict classification is the basic part of understanding the concept of conflicts. Given the importance of conflict classification as a form of conflict resolution control, several researchers have developed models for this goal. In developing the model, we set the following requirements:

- The model must provide a measure of confidence or confidence level of conflicting agents for each conflict situation, which allows comparison between conflicting agents.

- The model must provide a ratio of conflict which detects the number of conflicting agents in both conflicting sides.

- The model must provide a disagreement degree by detecting the number of dissenting issues in each conflict situation.

Based on agents' confidence values, two types of conflict are determined:

- Strong Conflict (SC): When two agents conflict more than $50 \%$ of their decisions or their opinions $(>1)$.

- Weak Conflict (WC): When two agents conflict less than $50 \%$ of their decisions or opinions $(<=1)$

While previous works in the literature explored different types of conflict classification [3, 8, 9]. This work explores the conflict classification by considering the Conflict Ratio and Disagreement Degree in evaluating the conflict strength. There are three key questions:

- The ratio of conflict between agents,

- The number of agents in each conflict state,

- The number of dissenting issues in each conflict state.

Definition 4: A conflict ratio, $\mathrm{CR}$, is a ratio of conflicting agents to total number of agents. Each conflict state in CAS has conflict ratio can be represented as a low $(\mathrm{L})$ or high $(\mathrm{H})$,

- If the number of CAS $>50 \%$ of the number of $\mathrm{A} \rightarrow \mathrm{CR}$ is $\mathrm{H}$

- If the number of CAS $<=50 \%$ of the number of $\mathrm{A} \rightarrow$ $\mathrm{CR}$ is $\mathrm{L}$

Conflict Ratio Calculation Function (CRCF): This function calculates the ratio of conflicting agents with total number of agents in one of conflicting sets.

$$
\mathrm{CR}: \mathrm{CR} \rightarrow \mathrm{CI} / \mathrm{TI}
$$

Where:

$\mathrm{CI}$ : is a conflicting issues,

TI: is a total issues in the conflict state.

Definition 5: An agent opinion base (AOB), denoted as a pair, $\mathrm{AOB}=(\mathrm{A}, \mathrm{O})$, where $\mathrm{A}$ and $\mathrm{O}$ are finite sets of agents and agents' opinions, respectively. 
Definition 6: A dissenting issues, DI, are an issues that agents conflicts about, if there are two agents ai and aj conflict about issues I1 and I2, then DI $=\{\mathrm{I} 1, \mathrm{I} 2\}$

\section{CONFlict Resolution StRATEgy SElection METHOD}

In many multi-agent applications, the delay in conflict resolution can cause a system performance degradation, so, a fast conflict resolution is required [10]. If there is more than one proposal within the negotiation strategy, the number of required messages is much more than any other strategy, which makes reaching agreement state more complicated. If the message has high cost bandwidth, this makes negotiation a high cost strategy. If any conflict state among agents resolved by negotiation strategy that requires many messages, this may be lead to a heavy coordination state. Based on this introduction, there is a real need to include some strategy that ignores some unimportant conflict states or include submitting strategy to enhance conflict resolution method performance. Figure 3 shows the process flow of selecting a conflict resolution strategy.

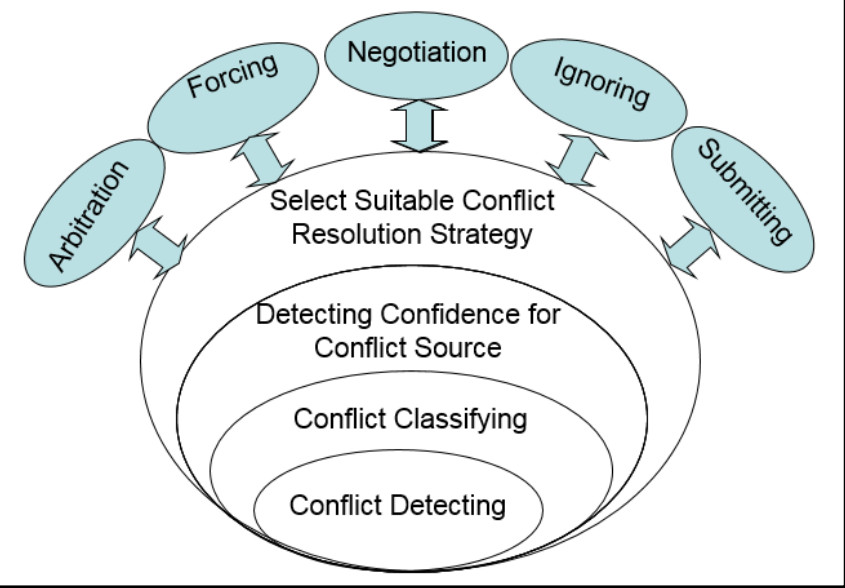

Fig. 3. Process Flow for Selecting an Appropriate Conflict Resolution Strategy

In the proposed method, the conflict strength and a confidence level of agents will be used for the selection of an appropriate conflict resolution strategy. The proposed model has several strategies as described below.

\section{A. Conflict Resolution Strategies}

Negotiation: considers the most popular strategy for resolving conflict in multi-agent systems. In negotiation strategy, it is assumed that all agents are rational and intelligent. This means the agents have the ability to make decisions that allowed it to reach their goals. In our proposed method, negotiation is selected when there is a high concern for both conflict parties; it corresponds to Compromising in social science, when one of the opponents is willing to yield. Negotiation is appropriate when both conflicting parties have equal confidence level, and neither party is strong enough to impose its decision or to resolve the conflict unilaterally [16]. Figure 4 shows the interactions among agents in negotiation strategy. The number of instances of each role that are required for operating the strategy can then be calculated.

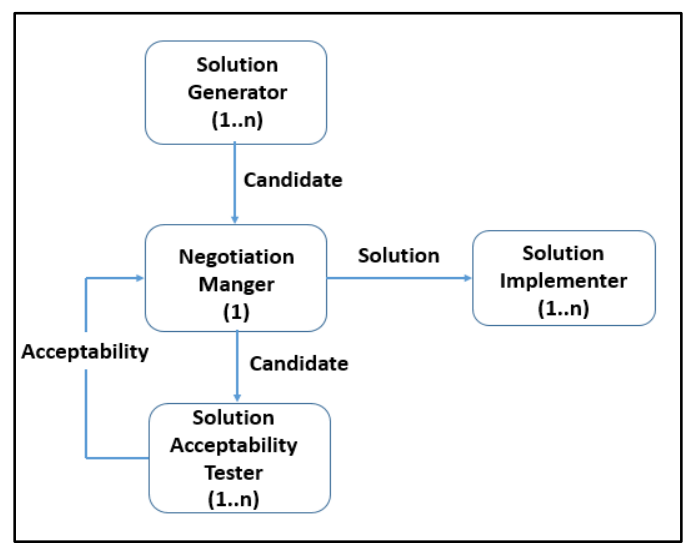

Fig. 4. Data Flow for Negotiation

Arbitration: corresponds to Delegation. Arbitration and mediation are processes in which conflicts are arbitrated or mediated by a third party that does not have control to modify the behaviours of the conflicting agents. In arbitration in contrast of mediation, the decision of the third party (Arbitrator) must be accepted by conflicting agents. The Arbitrator must have additional specifications like authority, complete knowledge and more solution-search capabilities than other agents $[17,18]$. This strategy is appropriate when a speedy decision domain requirement or a minimum number of messages is required. This strategy is appropriate when the agent disables to communicate with other agents. Figure 5 shows the interactions among agents in Arbitration strategy.

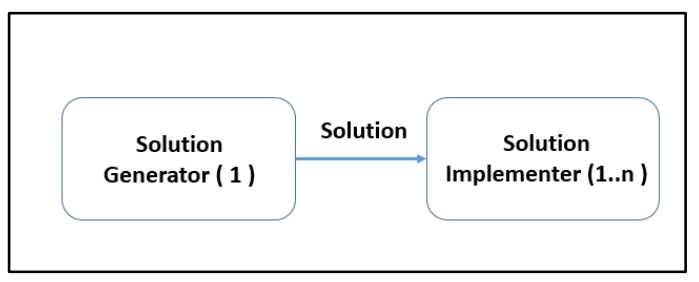

Fig. 5. Data Flow for Arbitration

Submitting: represents high concern for other agents and low concern for themselves. It corresponds to Subservience. In this case, there is no force, but the inducement between both sides. Zartman [16] argues in situations of perceived asymmetry, the stronger party tends to act exploitatively while the weaker acts submissively. Submitting strategy is useful when the conflict is weak and there is a clear difference between confidence levels of conflicting agents.

Ignoring: represents low concern for both conflict parties. This strategy similar to Withdrawing (Avoiding), that may happen when one of conflict's opposites does not pursue her/his own concerns [19]. One of the strategies that proposed in [20] is "Facilitation", that means the low level of conflict can be resolved by changing some variables. This strategy will be used when both conflicting agents have low level confidence, and the conflict strength between them is weak. In this case, ignoring the conflict give a good outcome than spending time and effort to resolve this conflict. It is appropriate if time and 
cost saving is one of the domain requirements. This strategy is inappropriate when one or both of the conflicting agents have a high confidence level, or when the conflict strength is strong.

Forcing: helps reduce the complexity by eliminating some options as part of a non-compensatory strategy [21]. This strategy is used when an agent cannot change its strategy [22]. It is similar to compromise.

Figure 6 shows the interactions among agents in Submitting, Ignoring and Forcing strategies.

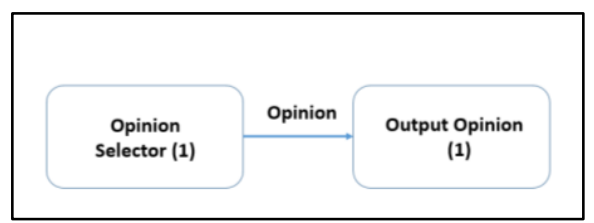

Fig. 6. Data Flow Diagram for Submitting, Ignoring and Forcing

\section{B. Strategies Characteristics Analysis}

We consider two main characteristics in the evaluation of conflict resolution strategies:

\section{1) The Number of Message for Each Strategy}

As shown in Figures 4 to 6, a number of messages are available for each strategy. Inter messages refer to each output/input event binding that is executed. The strategies represent the interactions between agents' roles. It is possible to calculate the number of messages and the data flow necessary to reach a solution through each strategy. The number of inter messages were calculated from the strategy representations using the following formulas:

- Number of Messages for Arbitration = No. of Solution Implementer

- No. of Messages in Negotiation $=\mathrm{P} *(1+2 *$ Solution Acceptability Testers No.) + No. of Solution Implementers

- Number of Messages for Forcing $=$ No. of Solution Implementer

- Number of Messages for Ignoring = No. of Solution Implementer

- Number of Messages for Submitting = No. of Solution Implementer

Where:

\section{P: Number of Proposals in Negotiation}

2) The Number of Time Ticks for Each Strategy

A time tick represents a consistent cut of the strategy execution history, where each role is executing a single reasoning process. The physical meaning for a time tick is that it is a synchronised point for coordinating modules' actions. All executions that may occur in a parallel fashion is synchronised among agents and their modules; one time tick corresponds to each role receiving an event, processing it, and outputting an event. These values cannot be used to directly compare the strategy performance, but rather to compare the behaviours exhibited by the strategies, such as scalability [1].

\section{THE CRSSA ARCHITECTURE}

Figure 7 depicts the architecture of Conflict Resolution Strategy Selector Agent (CRSSA). There are two main areas; the outer area represents the environment that contains conflicting agents set, and the inner area denotes the classifying conflict states, and selecting conflict resolution strategy. The Belief component represents an agent's belief that includes conflict states in the system, conflicting issues and confidence levels of agents that are collected from Evaluation Agent. The Desire component represents an agent's goal that includes selecting a strategy for resolving conflict states in the system. The Intention component includes classifying conflict states, and selecting a conflict resolution strategy.

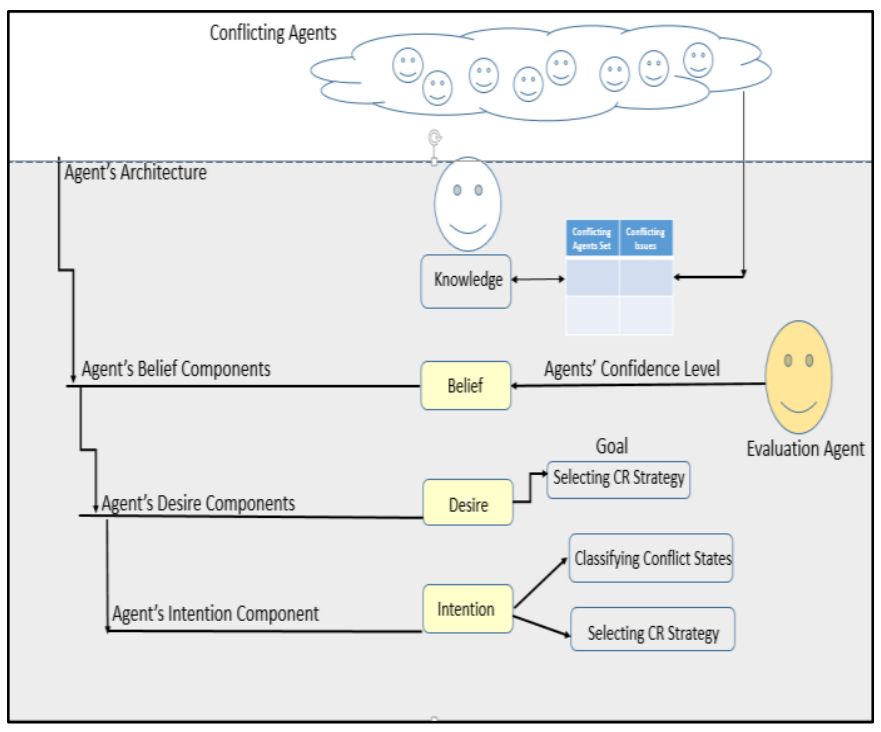

Fig. 7. The CRSSA Architecture

We hypothesise that the proposed ConfRSSM method will reduce both the number of messages and the number of time ticks for resolving all the conflict states in a given system.

\section{CONCLUSION AND FUTURE WORK}

Conflicts are likely to be the most critical parameters that are manifested through agents' communication in a distributed multi-agent system. This paper presented a novel approach to detect and select appropriate strategies for resolving conflicts in multi-agent environments according to: (1) The conflict strength between agents (weak conflict or strong conflict), and (2) The agent's ability (represented by its confidence level, decision-making ability and communication ability). We have also demonstrated that classifying conflicts is an important aspect for enhancing agents' interactions and cooperation. As part of the on-going work, we will simulate and validate the proposed ConfRSSM in the domain of Learning Management System (LMS). The simulation scenario includes four agents, each detects the student's learning style. The first agent represents a student, the second agent represents a student's parents (father or mother), the third agent represents a student's friend, and the fourth agent represents a student's lecturer. The four agents are expected to exploit the algorithmic steps proposed in ConfRSSM for selecting an appropriate conflict resolution strategy. 


\section{REFERENCES}

[1] Barber, K. S., Liu T. H. \& Han, D. C., "Strategic Decision-Making for Conflict Resolution in Dynamic Organized Multi-Agent Systems", A Special Issue of CERA Journal, 2000.

[2] Barber, K. S., Han, D. C., \& Liu, T. H., "Strategy Selection-Based MetaLevel Reasoning for Multi-Agent Problem Solving", Lecture Notes in Computer Science, pp. 269-283, 2001.

[3] Liu, T. H., Goel, A., Martin, C. E. \& Barber, K. S., "Classification and Representation of Conflict in Multi-agents Systems", Technical Report TR98-UT-LIPSAGENTS-01, The Laboratory for Intelligent Processes and Systems, University of Texas at Austin, 1998.

[4] Adler, M. R., Davis, A. B., Weihmayer, R., Worrest, R. W., "ConflictResolution Strategies for Nonhierarchical Distributed Agents", In Distributed Artificial Intelligence II, Gasser, L. and Huhns, M. N., Eds. London: Pitman Publishing, pp. 139-161, 1989.

[5] Giret, P. \& Noriega, A. P., "On Grievance Protocols for Conflict Resolution in Open Multi-Agent Systems", in Proceedings of the 44th Hawaii International Conference on System Sciences, 2011.

[6] Crawford, D., Bodine, R., "Conflict Resolution Education A Guide to Implementing Programs in Schools", Youth-Serving Organizations, and Community and Juvenile Justice Settings Program Report, 1996.

[7] Moraïtis, P., "A multi-criteria approach for distributed planning and conflict resolution for multi-agent systems", Retrieved from http://eblackcu.net/sandbox/items/show/8367, October 2013.

[8] Tessier, C., Chaudron, L., Muller, H., J., "Conflict agents, Conflict management in Multi Agent System", Vol. 1. Springer-Verlag, Berlin Heidelberg New York, 2000.

[9] Ghusoon Salim Basheer, Mohd Sharifuddin Ahmad, and Alicia Y.C. Tang, "A Framework for Conflict Resolution in Multi-agent Systems", 5th International Conference on Computational Collective Intelligence Technologies and Applications (ICCCI), Craiova, Romania, 11-13 September 2013. pp. 195-204.

[10] Jung, H., "Conflict Resolution Strategies and Their Performance Models for Large-scale Multiagent Systems", Diss. University of Southern California, 2013.

[11] Thomas, K., "Conflict and Conflict Management: Reflections and Update, Journal of Organizational Behavior”, 13(3), 1992, pp. 265-274.
[12] Ghusoon Salim Basheer, Mohd Sharifuddin Ahmad, Alicia Y.C. Tang, Sabine Graf, "Certainty, Trust and Evidence: Towards an Integrative Model of Confidence in Multi-agent Systems", Computers in Human Behavior, Volume 45, Elsevier, April 2015, Pages 307-315.

[13] Ghusoon Salim Basheer, Mohd Sharifuddin Ahmad, Alicia Y. C. Tang, "A Conflict Classification and Resolution Modeling in Multi-agent Systems", Encyclopedia of Information Science and Technology (3rd Ed.), DOI: 10.4018/978-1-4666-5888-2.ch685.

[14] Sugawara, T., "Emergence of Conventions in Conflict Situations in Complex Agent Network Environments (Extended Abstract)", in Proceedings of the 13th Inter-national Conference on Autonomous Agents and Multiagent Systems, France, 2014.

[15] Toshiharu, S., Kurihara, S., Hirotsu, T., Fukuda, K. \& Takada, T., "Conflict Estimation of Abstract Plans for Multi-agent Systems", In Proceedings of the 6th International Joint Conference on Autonomous Agents and Multi-agent Systems, 2007, ACM, pp. 125.

[16] Zartman, W. (1997). The Structuralism Dilemma in Negotiation. Research Group in International Security.

[17] Ioannidis, Y. E. and Sellis, T. K., "Conflict Resolution of Rules Assigning Values to Virtual Attributes”, In Proceedings of the 1989 ACM International Conference on the Management of Data, Portland, Oregon, 1989, pp 205-214.

[18] Ephrati, E. \& Rosenschein, J. S., "The Clarke Tax as a Consensus Mechanism among Automated Agents", In Proceedings of the Proceedings of the Ninth Conference on Artificial Intelligence, 1991, pp. 173-178.

[19] Hazleton, M., “Conflict Management Techniques”, Copyright, Human Metrics, 2013.

[20] Chih-Yao, L., "Multi-agent Conflict Coordination Using Game Bargain", Information Technology Journal 7.2, 2008, pp. 234-244.

[21] Helge G., "Decision-Making Strategies and Self-Regulated Learning: Fostering Decision-Making Competence in Education for Sustainable Development", PhD Thesis, der Georg-August-Universität Göttingen, 2011.

[22] Curwin, J., \& Slater, R., "Quantitative Methods for Business Decisions", Cengage Learning EMEA, London, 2007. 